\title{
Growth of protein crystals in high-strength hydrogels with the dialysis membrane
}

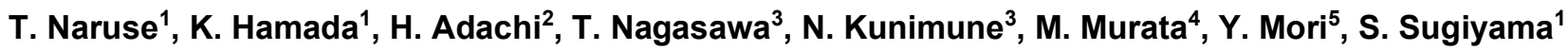 \\ ${ }^{1}$ Faculty of Science \& Technology, Kochi University, Kochi 780-8520, Japan, \\ ${ }^{2}$ SOSHO Inc., Osaka 565-0871, Japan, \\ ${ }^{3}$ Kunimune Corp., Osaka 532-0004, Japan, \\ ${ }^{4}$ Graduate School of Science, Osaka University, \\ ${ }^{5}$ Graduate School of Engineering, Osaka University \\ ssugiyama@kochi-u.ac.jp
}

High-throughput protein X-ray crystallography offers an unprecedented opportunity to facilitate drug discovery. The most reliable approach is to determine the three-dimensional (3D) structure of the protein-ligand complex by soaking the ligand in apo-crystals, but many lead compounds are not readily water-soluble. Such lead compounds must be dissolved in concentrated organic solvents such as DMSO. Therefore, to date, it has been impossible to produce crystals of protein-ligand complexes by soaking in apo-crystals, because protein crystals dissolve immediately upon soaking in concentrated organic solvents containing lead compounds. The problem arises from the influence of osmotic shock on crystal packing during soaking.

Protein crystals grown in hydrogel allow us to prevent serious damage to the crystals caused by soaking in high-concentration organic solvents, producing crystals of complexes between the target protein and poor water-soluble compounds by soaking in it. We previously reported the high-strength hydrogel method [1-4], but obstacles remain for general versatility. To overcome this difficulty, we devised an improved method for diffusing proteins into the pre-solidified hydrogel [5]. This study established a new crystallization method that prevents high-temperature damage to proteins. This method offers a technique to osmose the protein from the top of a hydrogel layer and recover its crystals with the precipitant on the bottom of the hydrogel layer by using a plate with a dialysis membrane. This study concentrated on the protein crystallization in hydrogels, but the results indicate that this method will be applicable to various proteins because it can always be operated at a low temperature.

[1] Sugiyama et al. (2012) J. Am. Chem. Soc., 134, 5786-5789.

[2] Sugiyama et al. (2013) Cryst. Growth Des., 13, 1899-1904.

[3] Sugiyama et al. (2015) CrystEngComm, 17, 8064-8071.

[4] Maruyama et al. (2016) J. Cryst. Growth, 452, 172-178.

[5] Sugiyama et al. (2021) Jpn. J. Appl. Phys., 60, No.015506.

\section{Keywords: high-strength hydrogels; protein crystallization; dialysis membrane}

This work was supported by JST A-STEP Grant Number JPMJTM19DC, JSPS KAKENHI Grant Number 19K06588, and JST ERATO Grant Number JPMJER1005, Japan. 